Commun. Fac. Sci. Univ. Ank. Series $A_{1}$ V. 37 , pp. $9-15$ (1988)

\title{
ON THE GAUSSIAN AND MEAN CURVATURES OF PARALLEL HYPERSURFACES
}

M. KEMAL SAĞEL and H. H. HACISALIHOĞLU

Department of Mathematics, Ankara University, Ankara, Turkey

\section{ABSTRACT}

In this study, we give a generalization of the higher order Gaussian curvature function and the mean curvature function about the parallel surfaces in $E^{3}$, for $E^{n+1}$.

\section{BASIC CONCEPTS}

DEFINITION 1: Let $M_{1}$ and $M_{2}$ are two hypersurfaces in $E^{n+1}$, with unit normal vector $N_{1}$ of $M_{1}$

$$
N_{1}=\sum_{i=1}^{n_{+1}} a_{i} \frac{\partial}{\partial x_{i}}
$$

Where each $a_{1}$ is a $C^{\infty}$ function on $M_{1}$. If there is a function $f$, from $M_{1}$ to $M_{2}$ such that

$$
\begin{aligned}
\mathbf{f}: \mathrm{M}_{1} \longrightarrow \mathrm{M}_{2} & \longrightarrow \mathrm{f}(\mathrm{P})=\left(\mathrm{p}_{1}+\mathrm{ra}_{1}(\mathrm{P}), \ldots, \mathrm{p}_{\mathrm{n}+1}+\mathbf{r a}_{\mathrm{n}+1}(\mathrm{P})\right) . \\
\mathrm{P} & \longrightarrow
\end{aligned}
$$

Then $M_{2}$ is called a parallel hypersurfaces of $M_{1}$, where $r \in R$ [1].

DEFINITION 2: Let $M$ be a hypersurface in $E^{n+1}$ and $S$ denotes the shape operator on $M$, at $P \in M$. The function $H$ defined by

$$
\begin{aligned}
\mathbf{H}: \mathbf{M} & \longrightarrow \mathbf{R} \\
\mathbf{P} & \longrightarrow \mathrm{H}(\mathrm{P})=\mathrm{tz}_{\mathrm{z}} \mathrm{P}(\mathrm{P})
\end{aligned}
$$

is called the mean curvature function of $M$ and the real number $H(P)$ is called mean curvature of $M$ at the point $P$ [3].

DEFINITION 3: Let $M$ be a hypersurface in $E^{n+1}$ and $S$ denotes the shape operator on $M$, at $P \in M$. The function $K$ defined by 


$$
\begin{aligned}
\mathbf{K}: \mathbf{M} & \longrightarrow \mathrm{R} \\
\mathrm{P} & \longrightarrow \mathrm{K}(\mathrm{P})=\operatorname{det} \mathrm{S}(\mathrm{P})
\end{aligned}
$$

is called the Gaussian curvature function of $M$ and the $K(P)$ is called Gaussian curvature of $M$ at the point $P$ [3].

DEFINITION 4: Let $M$ be a hypersurface in $E^{n+1}$ and $T_{M}(P)$ be a tangent space on $M$, at $P \in M$ : If $S_{P}$ denotcs the shape operator on $M$, at $\mathbf{P} \in \mathbf{M}$, then

$$
\mathrm{S}_{\mathrm{P}}: \mathrm{T}_{\mathrm{M}}(\mathrm{P}) \longrightarrow \mathrm{T}_{\mathrm{M}}(\mathrm{P})
$$

is a linear mapping. If we denote the characteristic values by $\lambda_{1}, \lambda_{2}, \ldots, \lambda_{n}$ and the corresponding characteristic vectors by $x_{1}, x_{2}, \ldots, x_{n}$ of $S_{P}$ then $\lambda_{1}, \lambda_{2}, \ldots, \lambda_{n}$ are the principal curvatures and $x_{1}, x_{2}, \ldots, x_{n}$ are the principal directions of $M$, at $P \in M$. On the other hand, if we use the notations

$$
\begin{aligned}
K_{1}\left(\lambda_{1}, \lambda_{2}, \ldots, \lambda_{n}\right) & =\sum_{i=1}^{n} \lambda_{i} \\
K_{2}\left(\lambda_{1}, \lambda_{2}, \ldots, \lambda_{n}\right) & =\sum_{i<j}^{n} \lambda_{i} \lambda_{j} \\
K_{3}\left(\lambda_{1}, \lambda_{2}, \ldots, \lambda_{n}\right) & =\sum_{i<j<t}^{n} \lambda_{i} \lambda_{j} \lambda_{t} \\
& \cdot \\
& \cdot \prod_{n}^{n} \lambda_{i}
\end{aligned}
$$

then the characteristic polinomial of $S(P)$ becomes

$$
\mathbf{P}_{\mathrm{S}(\mathrm{P})}(\lambda)=\lambda^{\mathrm{n}}+(-\mathbf{1}) \mathbf{K}_{1} \AA^{\mathrm{n}^{-1}}+\cdots+(-1)^{\mathrm{n}} \mathbf{K}_{\mathrm{n}}
$$

and $K_{i}, 1 \leq i \leq n$ are uniquely determined, where the functions $K_{i}$ are called the higher ordered Gaussian curvatures of the hypersurface M [4], [5].

THEOREM 1: Let $f$ be a mapping from $M$ to $M_{r}$ and $M_{r}$ be a parallel hypersurface of $M$. Then $f$ preserves the principal curvature directions [2] THEOREM 2: Let $M_{r}$ be a parallel surface of the surface $M \subset E^{3}$. Let the Gaussian curvature and mean curvature of $M$ be denoted by $K$ and $H$ at $P \in M$, respectively and the Gaussian curvature and mean curvature of $M_{r}$ be denoted by $K_{r}$ and $H_{r}$ at $f(P) \in M_{r}$, respectively. Then we know that 


$$
\mathbf{K}_{\mathrm{r}}=\frac{\mathbf{K}}{1+\mathbf{r} \mathbf{H}+\mathbf{r}^{2} \mathbf{K}}
$$

and

$$
\mathbf{H}_{\mathrm{r}}=\frac{\mathbf{H}+2 \mathbf{r} \mathbf{K}}{\mathbf{l}+\mathbf{r} \mathbf{H}+\mathbf{r}^{2} \mathbf{K}}
$$

[2].

\section{GENERALIZED THEOREMS}

THEOREM 1: Let $M$ be a hypersurface of $E^{n+1}$ and $K_{1}, K_{2}, \ldots, K_{n}$ are the highor order Gaussian curvatures and $\mathrm{k}_{1}, \mathrm{k}_{2}, \ldots, \mathrm{k}_{\mathrm{n}}$ the principal curvatures at the point $P \in M$.

Let us define a function

$$
\Phi: \mathrm{M} \longrightarrow \mathrm{R}
$$

such that

$$
\mathrm{P} \longrightarrow \Phi(\mathrm{P})=\Phi\left(\mathbf{r}, \mathrm{k}_{1}, \mathrm{k}_{2}, \ldots, \mathrm{k}_{\mathrm{n}}\right)
$$

$$
=\prod_{\mathbf{i}=1}^{\mathrm{n}}\left(1+\mathrm{rk}_{\mathbf{i}}\right)
$$

$\Phi\left(\mathbf{r}, \mathbf{k}_{1}, \mathbf{k}_{2}, \ldots, \mathbf{k}_{\mathrm{n}}\right)=1+\mathbf{r} \sum_{\mathrm{i}=1}^{\mathrm{n}} \mathbf{k}_{\mathrm{i}}+\mathrm{r}^{2} \sum_{\mathrm{i}<\mathrm{j}}^{\mathrm{n}} \mathbf{k}_{\mathrm{i}} \mathrm{k}_{\mathrm{j}}+\ldots$

$$
+\mathbf{r}^{\mathbf{n}} \prod_{\mathbf{i}=1}^{\mathrm{n}} \mathbf{k}_{\mathbf{i}}
$$

or

$\Phi\left(\mathrm{r}, \mathrm{k}_{1}, \mathbf{k}_{2}, \ldots, \mathbf{k}_{\mathrm{n}}\right)=1+\mathbf{r} \mathbf{K}_{1}+\mathrm{r}^{2} \mathbf{K}_{2}+\ldots+\mathbf{r}^{\mathrm{n}} \mathbf{K}_{\mathrm{n}}$,

where $r \in R$ is given in Definition 1 .

PROOF: We prove the theorem by induction method.

For $\mathbf{n}=1$, the theorem holds.

Actually,

$$
\begin{aligned}
& \Phi\left(\mathrm{r}, \mathrm{k}_{1}, \mathrm{k}_{2}, \ldots, \mathrm{k}_{\mathrm{n}}\right)=\prod_{\mathrm{i}=1}^{1}\left(\mathrm{l}_{\mathrm{i}}+\mathrm{rk}_{\mathbf{i}}\right) \\
& =1+\mathrm{r} \mathrm{k}_{1} \\
& =1+\mathbf{r} \cdot \sum_{\mathbf{i}=1}^{1} \mathrm{k}_{\mathrm{i}} \\
& =1+\mathrm{rK}_{1} \text {. }
\end{aligned}
$$


now Suppose that the theorem holds for $n=p$ and show that it is true for $n=p+l$ :

$$
\begin{aligned}
\Phi\left(\mathbf{r}, \mathbf{k}_{1}, \mathbf{k}_{2}, \ldots, \mathbf{k}_{\mathrm{n}}\right)= & \prod_{\mathbf{i}=1}^{\mathrm{p}}\left(\mathbf{l}+\mathbf{r} \mathbf{k}_{\mathbf{i}}\right) \\
= & \mathbf{l}+\mathbf{r} \sum_{\mathbf{i}=1}^{\mathrm{p}} \mathbf{k}_{\mathbf{i}}+\mathbf{r}^{2} \sum_{\mathbf{i}<\mathbf{j}}^{\mathbf{p}} \mathbf{k}_{\mathbf{i}} \mathbf{k}_{\mathbf{j}}+\ldots \\
& \quad+\mathbf{r} \prod_{\mathbf{i}=1}^{\mathrm{p}} \mathbf{k}_{\mathbf{i}} \\
& =1+\mathbf{r} \mathbf{K}_{1}+\mathbf{r}^{2} \mathbf{K}_{2}+\ldots+\mathbf{r}^{\mathbf{p}} \mathbf{K}_{\mathbf{p}} .
\end{aligned}
$$

For $\mathbf{n}=p+1$, we have

$$
\begin{aligned}
& \Phi\left(\mathbf{r}, \mathbf{k}_{1}, \mathbf{k}_{2}, \ldots, \mathbf{k}_{\mathrm{n}}\right)=\prod_{\mathrm{i}=1}^{\mathrm{p}+1}\left(\mathbf{l}+\mathbf{r k}_{\mathbf{i}}\right) \\
& =\left[\prod_{\mathbf{i}=1}^{p}\left(1+\mathbf{r k}_{\mathbf{i}}\right)\right]\left(1+\mathbf{r k}_{\mathbf{p}_{+1}}\right) \\
& =\left(1+r \sum_{i=1}^{p} k_{i}+r^{2} \sum_{i<j}^{p} k_{i} k_{j}+\ldots\right. \\
& \left.+\mathbf{r}^{\mathrm{p}} \prod_{\mathbf{i}=1}^{\mathrm{p}} \mathbf{k}_{\mathbf{i}}\right)\left(1+\mathbf{r k}_{\mathrm{p}+1}\right) \\
& =1+\mathbf{r}\left(\sum_{i=1}^{p} k_{i}+k_{p+1}\right)+r^{2}\left(\sum_{i<j}^{p} k_{i} k_{j}\right. \\
& \left.+k_{p+1} \sum_{i=1}^{p} k_{i}\right)
\end{aligned}
$$

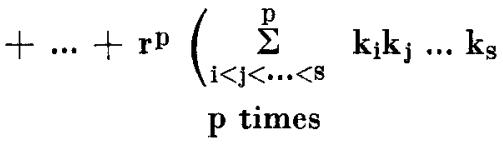

$$
\begin{aligned}
& \left.+\mathbf{k}_{\mathrm{p}+1} \underset{\mathrm{i}<\mathrm{j}<\cdots<1}{\stackrel{p}{\Sigma}} \mathbf{k}_{\mathbf{1}} \mathbf{k}_{\mathbf{j}} \ldots \mathbf{k}_{\mathbf{1}}\right) \\
& \text { p-1 times }
\end{aligned}
$$




$$
\begin{aligned}
& +\mathbf{r}^{p+1} \mathbf{k}_{\mathbf{p}+1} \prod_{\mathbf{i}=1}^{p} \mathbf{k}_{\mathbf{i}} \\
& =1+\mathbf{r} \sum_{i=1}^{p_{+}+1} \mathbf{k}_{\mathbf{i}}+\mathbf{r}^{2} \sum_{\mathbf{i}<j}^{p_{+1}} \mathbf{k}_{\mathbf{i}} \mathbf{k}_{\mathbf{j}} \\
& +\ldots+\mathbf{r}^{p} \sum_{\substack{i<j<\ldots<s \\
\mathbf{p} \text { times }}}^{p_{+1}} \mathbf{k}_{\mathbf{i}} \mathbf{k}_{\mathbf{j}} \ldots \mathbf{k}_{\mathrm{s}}+\mathbf{r}^{\mathrm{p}+1} \prod_{\mathbf{i}=1}^{\mathrm{p}_{+1}} \mathbf{k}_{\mathbf{i}} \\
& =1+\mathbf{r} \mathbf{K}_{1}+\mathbf{r}^{2} \mathbf{K}_{2}+\ldots+\mathbf{r}^{p} \mathbf{K}_{\mathbf{p}}+\mathbf{r}^{\mathbf{p}+1} \mathbf{K}_{\mathbf{p}_{+} 1} \text {, }
\end{aligned}
$$

which proves the theorem.

THEOREM 2: Let $M_{r}$ be a hypersurface to the parallel hypersurface $\mathbf{M}$ of $\mathbf{E}^{\mathbf{n}+1} \cdot \mathbf{K}_{1}, \mathbf{K}_{2}, \ldots, \mathbf{K}_{\mathrm{n}}$ denote the higher order Gaussian curvatures of $M$, at $P \in M$. $K_{r}$ and $H_{r}$ generalized Gaussian and mean curvature of $M_{r}$, at the point $f(P)$.

Suppose the function $\Phi: \mathbf{M} \longrightarrow \mathbf{R}$

$$
\begin{aligned}
\mathbf{P} \longrightarrow \Phi(\mathrm{P}) & =\Phi\left(\mathbf{r}, \mathbf{k}_{1}, \mathbf{k}_{2}, \ldots, \mathbf{k}_{\mathrm{n}}\right) \\
& =\prod_{\mathrm{i}=1}^{\mathrm{n}}\left(\mathbf{l}+\mathbf{r k}_{\mathrm{i}}\right) .
\end{aligned}
$$

Then we have

$$
\mathbf{K}_{\mathrm{r}}=\frac{\frac{\partial^{\mathbf{n}} \Phi\left(\mathbf{r}, \mathbf{k}_{1}, \mathbf{k}_{2}, \ldots, \mathbf{k}_{\mathbf{n}}\right)}{(\partial \mathbf{r})^{\mathbf{n}}}}{(\mathbf{n} !) \Phi\left(\mathbf{r}, \mathbf{k}_{1}, \mathbf{k}_{2}, \ldots, \mathbf{k}_{\mathbf{n}}\right)}
$$

and

$$
\mathbf{H}_{\mathbf{r}}=\frac{\frac{\partial \Phi\left(\mathbf{r}, \mathbf{k}_{1}, \mathbf{k}_{2}, \ldots, \mathbf{k}_{\mathrm{n}}\right)}{\partial \mathbf{r}}}{\Phi\left(\mathbf{r}, \mathbf{k}_{1}, \mathbf{k}_{2}, \ldots, \mathbf{k}_{\mathrm{n}}\right)} .
$$

PROOF: Let $X_{1}, X_{2}, \ldots, X_{n}$ are the principal directions and $k_{1}, k_{2}, \ldots, k_{n}$ principal curvatures of $M$, at $P \in M$.

Let $f_{*}\left(X_{1}\right), f_{*}\left(X_{2}\right), \ldots, f_{*}\left(X_{n}\right)$ be principal directions at the point $f(P)$ of $M_{r}$ then we know that [2].

$$
\mathrm{S}_{\mathrm{r}}\left(\mathrm{f}_{*} \mathrm{X}_{1}\right)=\frac{\mathrm{k}_{1}}{1+\mathrm{rk}_{1}} \mathrm{f}_{*} \mathrm{X}_{1}
$$




$$
\begin{aligned}
\mathrm{S}_{\mathrm{r}}\left(\mathrm{f}_{*} \mathrm{X}_{2}\right) & =\frac{\mathbf{k}_{2}}{1+\mathrm{rk}_{2}} \mathrm{f}_{*} \mathrm{X}_{2} \\
& \cdot \\
& \cdot \\
\mathrm{S}_{\mathrm{r}}\left(\mathrm{f}_{*} \mathrm{X}_{\mathrm{n}}\right) & =\frac{\mathbf{k}_{\mathrm{n}}}{1+\mathrm{rk}_{\mathrm{n}}} \mathrm{f}_{*} \mathrm{X}_{\mathrm{n}} .
\end{aligned}
$$

Then, we know that, the shape operator of $M_{r}$ is

$$
\mathrm{S}_{\mathrm{r}}=\left[\begin{array}{cccc}
\frac{\mathrm{k}_{1}}{1+\mathrm{rk}_{1}} & 0 & \ldots & 0 \\
0 & \frac{\mathrm{k}_{2}}{1+\mathrm{rk}_{2}} & \cdots & 0 \\
\vdots & \vdots & & \vdots \\
\dot{0} & \dot{0} & \cdots & \frac{\mathrm{k}_{\mathrm{n}}}{1+\mathrm{rk}_{\mathrm{n}}}
\end{array}\right]
$$

and

$$
\begin{aligned}
& \mathbf{K}_{\mathrm{r}}=\operatorname{det} \mathrm{S}_{\mathbf{r}} \\
& =\frac{\mathbf{k}_{1}}{1+\mathbf{r k}_{1}} \cdot \frac{\mathbf{k}_{2}}{\mathbf{l}+\mathbf{r k}_{2}} \ldots \frac{\mathbf{k}_{\mathrm{n}}}{1+\mathbf{r k}_{\mathrm{n}}} \\
& =\frac{\mathbf{k}_{1} \cdot \mathbf{k}_{1} \ldots \mathbf{k}_{\mathbf{n}}}{\prod_{\mathrm{i}=1}^{\mathrm{n}}\left(\mathbf{1}+\mathbf{r k}_{\mathrm{i}}\right)} \\
& =\frac{\prod_{i=1}^{n} \mathbf{k}_{\mathbf{i}}}{\prod_{\mathbf{i}=1}^{\mathrm{n}}\left(1+\mathbf{r k}_{\mathbf{i}}\right)}
\end{aligned}
$$

and

$$
\Phi\left(\mathbf{r}, \mathrm{k}_{1}, \mathrm{k}_{2}, \ldots, \mathrm{k}_{\mathrm{n}}\right)=\prod_{\mathbf{i}=1}^{\mathrm{n}}\left(\mathbf{l}+\mathrm{rk}_{\mathrm{i}}\right)
$$

then 


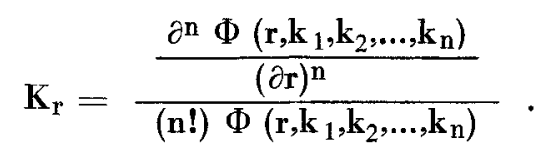

On the other hand

$$
\begin{aligned}
& \mathrm{H}_{\mathrm{r}}=\mathrm{Iz} \mathrm{S}_{\mathrm{r}} \\
& =\frac{\mathbf{k}_{1}}{1+\mathbf{r k}_{1}}+\frac{\mathbf{k}_{2}}{1+\mathbf{r k}_{2}}+\ldots+\frac{\mathbf{k}_{\mathbf{n}}}{1+\mathbf{r k}_{\mathrm{n}}} \\
& \mathbf{k}_{1} \prod_{i=2}^{n}\left(1+\mathbf{r k}_{\mathbf{i}}\right)+\left(1+\mathbf{r} \mathbf{k}_{1}\right) \mathbf{k}_{2} \prod_{i=3}^{n}\left(1+\mathbf{r k}_{i}\right)+\ldots+\mathbf{k}_{\mathbf{n}} \prod_{i=1}^{n_{-1}}\left(1+\mathbf{r k}_{\mathbf{i}}\right) \\
& \prod_{\mathbf{i}=1}^{\mathrm{n}}\left(1+\mathbf{r k}_{\mathbf{i}}\right)
\end{aligned}
$$

Since

$$
\Phi\left(\mathbf{r}, \mathbf{k}_{1}, \mathbf{k}_{2}, \ldots, \mathbf{k}_{\mathrm{n}}\right)=\prod_{\mathbf{i}=1}^{\mathrm{n}}\left(\mathbf{l}+\mathbf{r k}_{\mathrm{i}}\right),
$$

we get that

$$
\mathbf{H}_{\mathbf{r}}=\frac{\frac{\partial \Phi\left(\mathbf{r}, \mathbf{k}_{1}, \mathbf{k}_{2}, \ldots, \mathbf{k}_{\mathbf{n}}\right)}{\partial \mathbf{r}}}{\Phi\left(\mathbf{r}, \mathbf{k}_{1}, \mathbf{k}_{2}, \ldots, \mathbf{k}_{n}\right)}
$$

The case $n=2$ of this theorem reduces to the result which is given in [2].

\section{REFERENCES}

1 HACISALIHOĞLU, H.H., 1982, Diferensiyel Geometri. Inönü Üniversitesi Fen-Edebiyat Fakiiltesi Yaymlan, Mat. no: 2.

2 HICKS, NOEL J., 1963. Connexion Preserving, Conformal and Parallel maps. Reccived january 3o. $p p$ : 295-302.

3 HICKS, NOEL, J., 1974. Notes on Differential Geometry. Von Nostrand Reinhold Company, London.

4 ÖZDAMAR, E-HACISALHHOGLU, H.H., 1977. Higher Order Gaussian Curvatures and Fundamental Forms. Journal of the Fac. Sci. of the K.T.U.Vol. 1. Fase 9. pp: 99-116 series. MA : Mathematics

5 KOBAYASHI, S-NOMIZU, K., 1969. Foundations of Differential Geometry. John Wiley Sons, Inc. Volume II. pp : 23-33. 